\title{
Erratum: Electroluminescent study of polarization changes in lead magnoniobate crystals in pulsed electric fields [Phys. Solid State 39, 298 (February 1997)]
}

N. N. Kraĭnik, S. A. Popov, and S. A. Flerova

Fiz. Tverd. Tela (St. Petersburg) 39 (May 1997)

[S1063-7834(97)04105-1]

The caption to Fig. 1 should read: The scale along the $t$ axis (in $\mu \mathrm{s}$ ) is a-3.0, b-5.0, c -15 .

\section{Erratum: Polaritons in layered media and size quantization [Phys. Solid State 38, 1885 (November 1996)]}

O. W. Gorya

Fiz. Tverd. Tela (St. Petersburg) 39 (May 1997)

Through the author's oversight expression (7) was given incorrectly. The correct expression (7) should be

$$
\frac{m_{1}}{m_{2}} \sqrt{\frac{m_{2} E}{m_{1}(V-E)}}=\left\{\begin{array}{l}
-\tan \left(k_{2} d_{2} / 2\right) \\
\cot \left(k_{2} d_{2} / 2\right)
\end{array}\right.
$$

Therefore expression (8) should be $\varepsilon_{1} / \varepsilon_{2}=m_{1} / m_{2}$. Then substituting Eq. (8) into (6) does not give an expression identical to the dispersion relation given in Ref. 6, p. 32, but in that monograph this relation was published with a misprint. Since the result $\varepsilon_{n} \rightarrow m_{n}$ does indeed follow from a comparison of the squares of the phase velocities of the electromagnetic waves and the de Broglie waves, the main conclusions of the paper, including the prediction that the superlattice is absolutely opaque, remain in force. 